\title{
Fine Grain Barium Ferrite Thin Films With Perpendicular C-axis Orientation
}

\author{
Yingjian CHEN, James A. BAIN, and Mark H. KRYDER \\ Data Storage Systems Center, Carnegie Mellon University, Pittsburgh, PA 15213
}

\begin{abstract}
Perpendicular barium hexaferrite thin films were fabricated using ex-situ and in-situ annealing. Our experimental results suggest that there are two different types of nucleation processes during the ex-situ annealing of the amorphous barium ferrite thin films. The nucleation within the film "bulk" tends to form crystallites with random c-axis orientations, whereas the nucleation at the interface(s) with the substrates or underlayers forms crystallites with the c-axis predominantly perpendicularly oriented. A Pt underlayer was shown to promote the interface nucleation which results in better perpendicular orientation. The barium ferrite films with Ba content higher than stoichiometry which were previously shown to have finer grain size, however, tend to grow grains of random orientation even on $\mathbf{P t}$ underlayers due to the much enhanced "bulk" nucleation. Nearly perfect perpendicular orientation can be achieved in the high $B a$ content films if the films are made to be thin $<300 \AA$, and a twostep annealing is used. The films using in-situ annealing showed self-epitaxial growth of grains with perpendicular orientation, which can also be improved by using a Pt underlayer.
\end{abstract}

Key words: barium hexaferrite, Pt underlayer, perpendicular recording, grain size

\section{INTRODUCTION}

Barium ferrite thin films are promising candidates for both longitudinal and perpendicular recording applications. They can be fabricated by sputter deposition with either in-situ or ex-situ annealing $[1,2]$. Barium ferrite films deposited with in-situ annealing often exhibit perpendicular orientation even when oxidized silicon substrates are used without an underlayer [1]. Various underlayers, such as $\mathrm{ZnO}$, were used in order to enhance the perpendicular orientation [3]. Barium ferrite films fabricated using ex-situ annealing generally exhibit random c-axis orientation when deposited on $\mathrm{SiO}_{2}$ substrates [2]. A Pt underlayer was shown to be effective in promoting perpendicular orientation [4]. The films previously studied had nearly stoichiometric composition. These films, despite their excellent c-axis perpendicular orientation, exhibit low coercivity ( $1500 \mathrm{Oe})$ and large grain size $(\sim$ $2000 \AA$ ), which would not meet the needs for high density recording applications.

It has been shown that barium ferrite films with $\mathrm{Ba}$ content higher than stoichiometry have high nucleation rates and lower growth rates during the post-deposition crystallization process [5]. As a result, much finer grain size was achieved in the high Ba content films. The films which are $10 \mathrm{wt} \%$ rich in $\mathrm{BaO}$ have a grain size of about $300 \AA$ and a coercivity of about 4500 5000 Oe, which appear promising for future high density recording applications.

In this study, effects of a $\mathrm{Pt}$ underlayer on the barium ferrite thin films of nearly stoichiometric composition and 10 $w t \% \mathrm{BaO}$ rich were investigated. It is shown that fine grain and high coercivity barium ferrite films with nearly perfect perpendicular c-axis orientation can be achieved.

\section{EXPERIMENTAL PROCEDURES}

Barium ferrite thin films were deposited from two targets of different $\mathrm{BaO}$ contents by rf diode sputtering in a Leybold $\mathrm{Z}-400$ sputtering system. The $\mathrm{BaO}$ contents in the films are 12 $w t \%$ and $23 \mathrm{wt} \%$. A sputtering power of $30 \mathrm{~W}$ at a rate of about $7.5 \AA / \mathrm{min}$, and $\mathrm{Ar} / \mathrm{O}_{2}$ gas with flow ratio of $67 / 12$ sccm were used for films with ex-situ or in-situ annealing. $\mathrm{Pt}$ underlayers were deposited using a sputtering power of 100 $W$ at a rate of $130 \AA / \mathrm{min}$ with $75 \mathrm{sccm}$ Ar flow. The Pt underlayers were $2000 \AA$ thick except when the effect of Pt thickness was being studied and varied from 200 to $2000 \AA$.

When in-situ heating was used, $\mathrm{SiO}_{2}$ substrates with or without $2000 \AA \mathrm{Pt}$ underlayers were silver pasted and dried on a heater stage in the sputtering chamber in order to ensure good thermal contact. The heater stage was then heated to 800 ${ }^{\circ} \mathrm{C}$ in vacuum and this temperature was maintained during the sputtering process.

The ex-situ annealed films were deposited at room temperature and annealed after deposition using a rapid thermal annealing (RTA) furnace. Since the high Ba content films are easier to crystallize, they were annealed at $790{ }^{\circ} \mathrm{C}$ for $60 \mathrm{~s}$. The low Ba content films were annealed at $810^{\circ} \mathrm{C}$ for $60 \mathrm{~s}$. Such annealing conditions were sufficient to fully crystallize these films and the saturation magnetization $M_{s}$ showed no increase upon further annealing. Some asdeposited films were annealed using a two-step annealing process [6]. They were homogenized at $600^{\circ} \mathrm{C}$ in a tube furnace for 10 hours before RTA.

The magnetic properties of the films were studied using an alternating gradient magnetometer (AGM). The out-of-plane torque curves were measured using a torque magnetometer at a field of $12000 \mathrm{Oe}$. The perpendicular anisotropy field $\mathrm{H}_{k}$ was calculated from the peak-to-peak torque values and saturation magnetization $M_{s}$ using $H_{k}=2 K_{u} / M_{s}$.

The crystalline structures of the films were characterized using X-ray diffraction.

An atomic force microscope was used to study the surface morphology of the barium ferrite films and the Pt underlayers.

\section{RESULTS}

\section{A. Ex-situ annealing}

The low Ba content barium ferrite films deposited directly onto $\mathrm{SiO}_{2}$ substrates are not ferromagnetic at thickness less than $500 \AA$. At $800 \AA$ or more, the films have similar in-plane and perpendicular magnetic properties, and the asymptotic values of $0.55 \sim 0.6$ and 2500 Oe were reached for squareness $\left(\mathrm{S}_{\mathrm{q}}\right)$ and coercivity $\left(\mathrm{H}_{\mathrm{c}}\right)$, respectively. The values of $\mathrm{S}_{\mathrm{q}}$ and $\mathrm{H}_{\mathrm{c}}$ were slightly higher in the perpendicular direction for the 500 $\AA$ thick film. The films with the two-step annealing have slightly lower $\mathrm{M}_{\mathrm{r}} \mathrm{t}, \mathrm{S}_{\mathrm{q}}$ and $\mathrm{H}_{\mathrm{c}}$ values. 
The films deposited on $2000 \AA \mathrm{Pt}$ underlayers became ferromagnetic even at $200 \AA$. At the thickness of $300 \AA, \mathrm{M}_{\mathrm{r}} \mathrm{t}$ is about $0.6 \mathrm{memu} / \mathrm{cm}^{2}$ in the perpendicular direction. It is about $2 \mathrm{memu} / \mathrm{cm}^{2}$ for the $1250 \AA$ thick film, which is about $25 \%$ higher than the film of the same thickness deposited on the $\mathrm{SiO}_{2}$ substrate. The values of $\mathrm{M}_{\mathrm{r}} \mathrm{t}$ are much greater in the perpendicular than in-plane direction, especially for thinner films. The values of $S_{\mathrm{q}}$ are over 0.9 in the perpendicular direction for the films of $300 \AA \sim 800 \AA$ as shown in Fig. 1 . They are less than 0.25 in-plane. Even higher perpendicular $S_{q}$ values than in-plane were found in the films using the twostep annealing. The $500 \AA$ film (A) using the two-step annealing, whose hysteresis loops are shown in Fig. 2a, has $S_{q}$ of nearly 1 in the perpendicular direction, but only 0.05 inplane. For the films which were $500 \AA$ or less in thickness, the two-step annealing also resulted in much reduced in-plane coercivity as seen in Fig. 3. The $1250 \AA$ thick films have less different in-plane and perpendicular $S_{q}$ and similar $H_{c}$ values as shown in Figs. 1 and 3. The values of $H_{c}$ also increase to over 2500 Oe for the $1250 \AA$ film using RTA only. Such an increase was not observed for the film of the same thickness, but which underwent the two-step annealing.

The high $\mathrm{Ba}$ content films deposited on $\mathrm{SiO}_{2}$ become ferromagnetic at the film thickness of $300 \AA$ or more. Similar in-plane and perpendicular magnetic properties were observed except in the $300 \AA$ films. In fact, in the $300 \AA$ film using the two-step annealing, the perpendicular and inplane $S_{q}$ are 0.84 and 0.1 , and $H_{c}$ are $1800 \mathrm{Oe}$ and $450 \mathrm{Oe}$, respectively. For the films which were $500 \AA$ or thicker, $S_{q}$ is in the range $0.5 \sim 0.6$ and $\mathrm{H}_{\mathrm{c}}$ is in the range $4000 \sim 4500 \mathrm{Oe}$.

The films with high $\mathrm{Ba}$ content deposited on $2000 \AA \mathrm{Pt}$ underlayers were weakly magnetic even at $200 \AA$ thickness. The films of $500 \AA$ or larger thickness have similar in-plane and perpendicular $M_{r} t, S_{q}$ and $H_{c}$ values as shown in Figs. 4 and 5, which show the thickness dependence of $S_{q}$ and $H_{c}$, respectively. $S_{q}$ is about $0.6 \sim 0.65$, and $H_{c}$ is about 4500 Oe for films of $500 \AA$ or more thickness. Much higher $S_{q}$ and $H_{c}$ were, however, observed in the perpendicular direction in the $300 \AA$ thick film (B) with the two-step annealing. This film, whose hysteresis loop is shown in Fig. $2 b$, has $S_{q}$ of $0.9, H_{c}$ of 4000 Oe and $M_{\mathrm{r}} \mathrm{t}$ of $0.4 \mathrm{memu} / \mathrm{cm}^{2}$ in the perpendicular

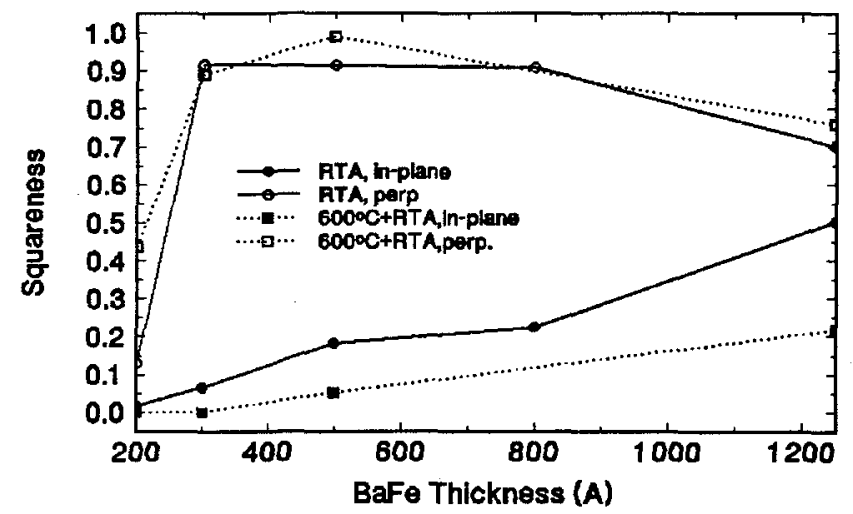

Fig. 1. Thickness dependence of the in-plane and perpendicular squareness of the low Ba content films deposited on Pt underlayers.

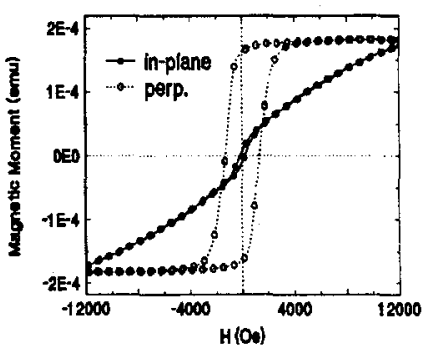

(a)

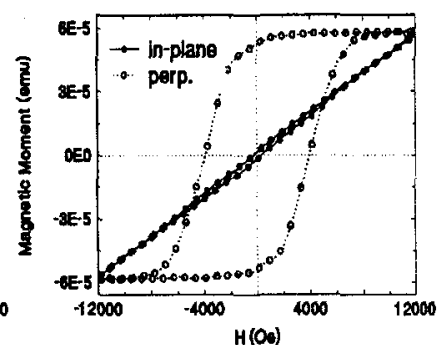

(b)
Fig. 2. The in-plane and perpendicular hysteresis loops of film $A$, a $500 \AA$ low Ba content film (a), and of film B, a $300 \AA$ high Ba content film (b). Both were deposited on $\mathrm{Pt}$ underlayer and ex-situ annealed using the twostep annealing.

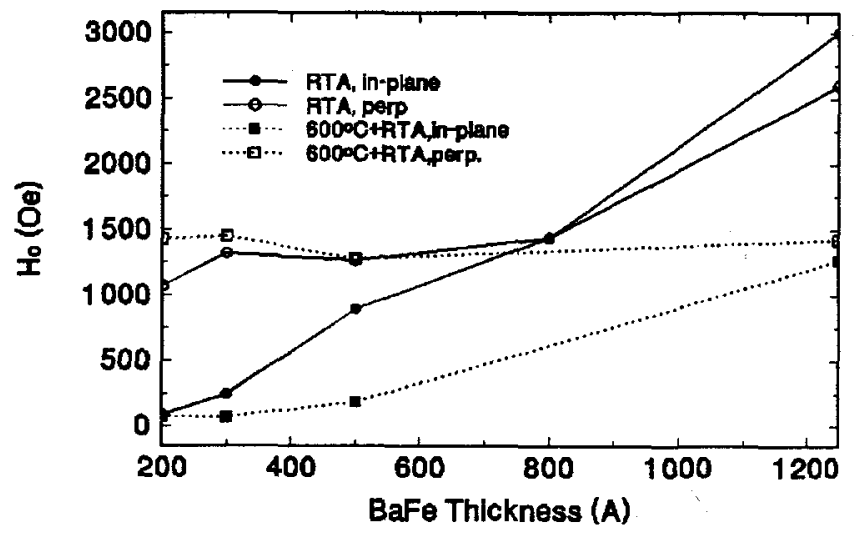

Fig. 3. Thickness dependence of the in-plane and perpendicular coercivity of the low $\mathrm{Ba}$ content films deposited on Pt underlayers.

direction; while the same quantities are almost zero in the inplane direction, indicating nearly perfect perpendicular orientation.

The values of $\mathrm{H}_{\mathrm{k}}$ measured from the torque are about 13000 Oe and 12000 Oe for films A and B, respectively. The values of $\mathrm{H}_{k}$ may also be taken as the field where the hard axis and easy axis loops intersect with each other as shown in Fig. 2. They are consistent with the torque measurements.

The X-ray diffraction patterns of films $\mathrm{A}$ and $\mathrm{B}$ are shown in Fig. 6. The predominant $(00 l)$ diffraction peaks indicate perpendicular c-axis orientation of in both films. Much weaker peaks were observed in film B with high $\mathrm{Ba}$ content, which is consistent with its reduced grain size.

The Pt underlayer thickness was also varied from $200 \AA$ to $2000 \AA$, while keeping the barium ferrite layer at the thickness of $500 \AA$. For the low Ba content films, the values of perpendicular $\mathrm{H}_{c}$, which are in the range of $1200 \sim 1400 \mathrm{Oe}$, are relatively independent of the Pt thickness. A gradual increase of the perpendicular $S_{q}$ from 0.9 to about 0.99 was observed as the Pt thickness was increased from $200 \AA$ to $2000 \AA$ in the films using the two-step annealing. The inplane $S_{q}$ decreased from 0.25 to 0.05 for the same films. The in-plane $\mathrm{H}_{c}$ also decreased from $1200 \mathrm{Oe}$ to $200 \mathrm{Oe}$, and showed a broad maximum when the Pt underlayer was $500 \AA$ thick. The in-plane $\mathrm{H}_{\mathrm{c}}$ showed a sharp maximum of $2000 \mathrm{Oe}$ at the Pt thickness of $300 \AA$ in the films using RTA only. The magnetic properties of the $500 \AA$ high $\mathrm{Ba}$ content films were 


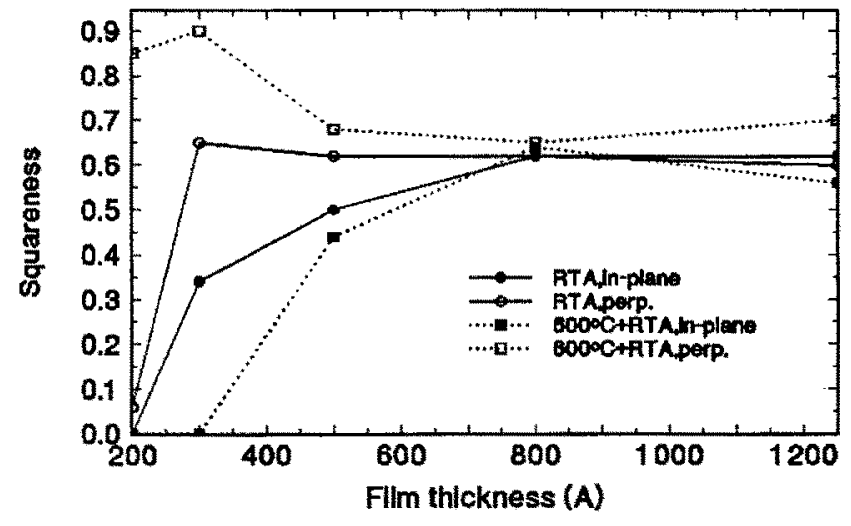

Fig. 4. Thickness dependence of the in-plane and perpendicular squareness of the high Ba content films deposited on Pt underlayers.

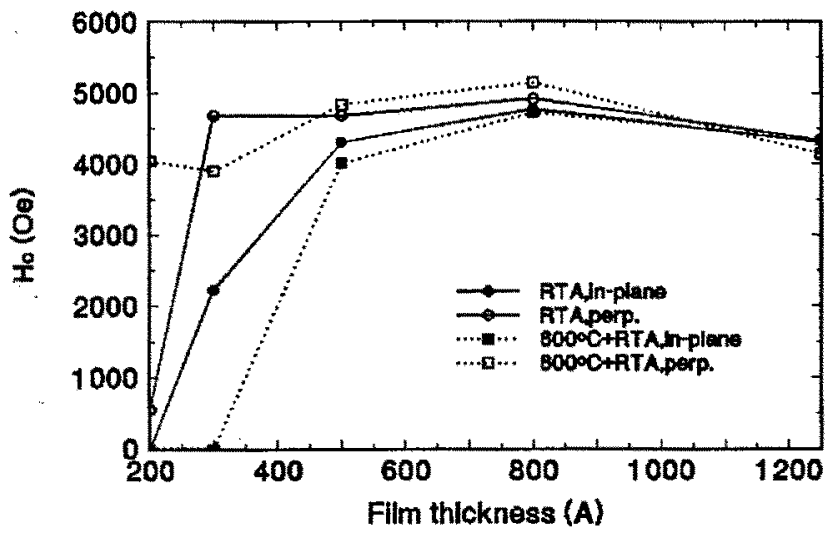

Fig. 5. Thickness dependence of the in-plane and perpendicular coercivities of the high Ba content films deposited on Pt underlayers.

found to be independent of the Pt underlayer thickness. Larger surface morphological features were observed by using an AFM in the thicker Pt underlayers which may be related to the larger Pt grain size.

\section{B. In-situ annealing}

Under in-situ annealing, the low Ba content films deposited on $\mathrm{SiO}_{2}$ substrates even without $\mathrm{Pt}$ underlayers have moderately higher perpendicular $S_{q}$ than in-plane $S_{q}$, as shown in Fig. 7. By using a Pt underlayer the $S_{q}$ in the

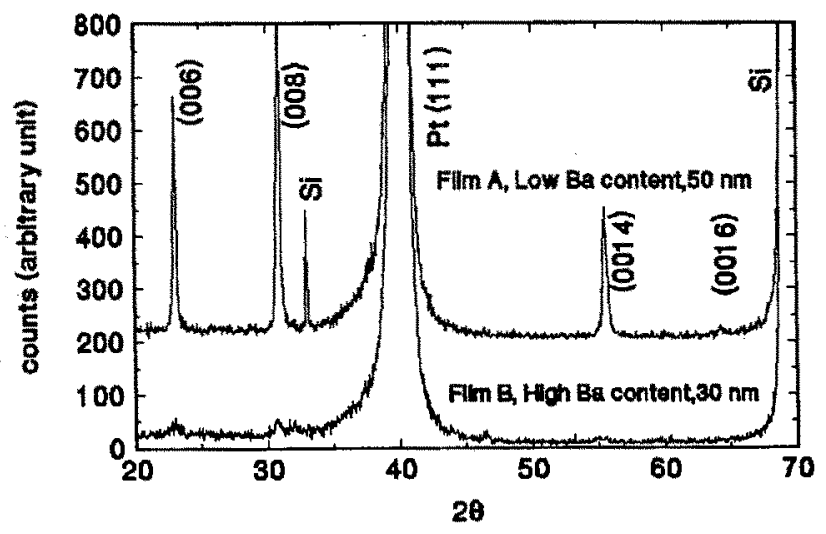

Fig. 6. X-ray diffraction patterns of film A and film B. perpendicular direction was enhanced. The magnetic properties of high $\mathrm{Ba}$ content films with in-situ annealing are quite similar to the low Ba content films as shown in Table I. The perpendicular $\mathrm{H}_{\mathrm{c}}$ of the thicker high $\mathrm{Ba}$ content films is about $3000 \mathrm{Oe}$, which is higher than the low Ba content films, but lower than the ex-situ annealed films.

The X-ray diffraction patterns as shown in Fig. 8 indicate much stronger $(00 l)$ peaks in the film on a Pt underlayer than on $\mathrm{SiO}_{2}$, both of which were $800 \AA$ thick, and in-situ annealed with low Ba content. Weaker $(00 l)$ peaks were found in thinner films. No other barium ferrite peaks were found. The XRD patterns of the in-situ annealed high $\mathrm{Ba}$ content films are quite similar to the low Ba content films.

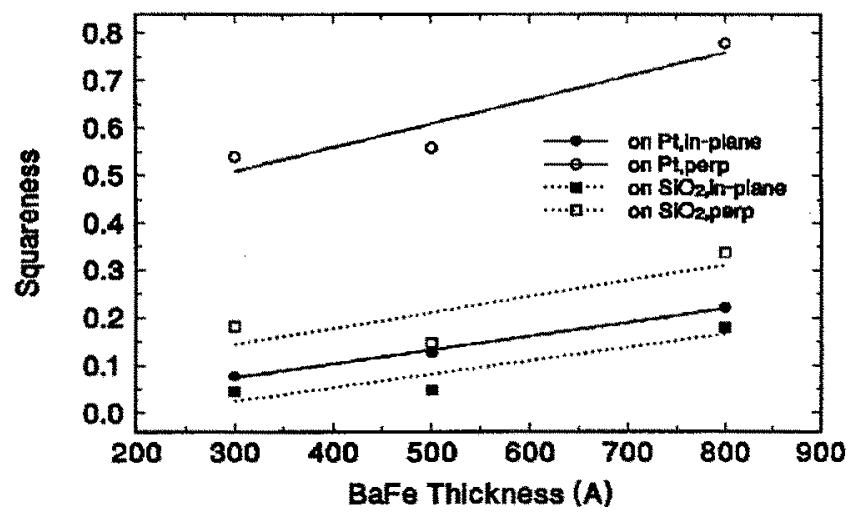

Fig. $7 \mathrm{ln}$-plane and perpendicular squareness of the in-situ annealed low Ba content films having the thickness of $300 \AA, 500 \AA, 800 \AA$.

TABLE I

MAGNETIC PROPERTIES OF IN-STTU ANNEALED FILMS ON Pt UNDERLAYERS

\begin{tabular}{|c|c|c|c|c|c|c|}
\hline & \multicolumn{3}{|c|}{ In-plane } & \multicolumn{3}{|c|}{ Perpendicular } \\
\hline $\begin{array}{l}\text { Ba content } \\
\text { Thickness }\end{array}$ & $\begin{array}{c}\mathrm{M}_{\mathrm{s}} \text { /area } \\
\left(\mathrm{memu} / \mathrm{cm}^{2}\right)\end{array}$ & $\mathrm{S}_{\mathrm{q}}$ & $\begin{array}{c}\mathrm{H}_{\mathrm{c}} \\
(\mathrm{Oe})\end{array}$ & $\begin{array}{c}\mathrm{M}_{s} / \mathrm{area} \\
\text { (memu/cm })\end{array}$ & $S_{q}$ & $\begin{array}{c}\mathrm{H}_{c} \\
\text { (De) }\end{array}$ \\
\hline low, $300 \AA$ & 0.53 & 0.078 & 418 & 0.50 & 0.539 & 2390 \\
\hline low, $500 \AA$ & 0.61 & 0.127 & 491 & 0.59 & 0.558 & 2520 \\
\hline $10 w, 800 \AA$ & 0.94 & 0.221 & 763 & 0.94 & 0.779 & 2040 \\
\hline high, $300 \AA$ & 0.60 & 0.136 & 423 & 0.55 & 0.154 & 1270 \\
\hline high, $500 \AA$ & 0.71 & 0.143 & 537 & 0.69 & 0.552 & 2920 \\
\hline high, $800 \AA$ & 0.90 & 0.222 & 1070 & 0.94 & 0.848 & 2900 \\
\hline
\end{tabular}

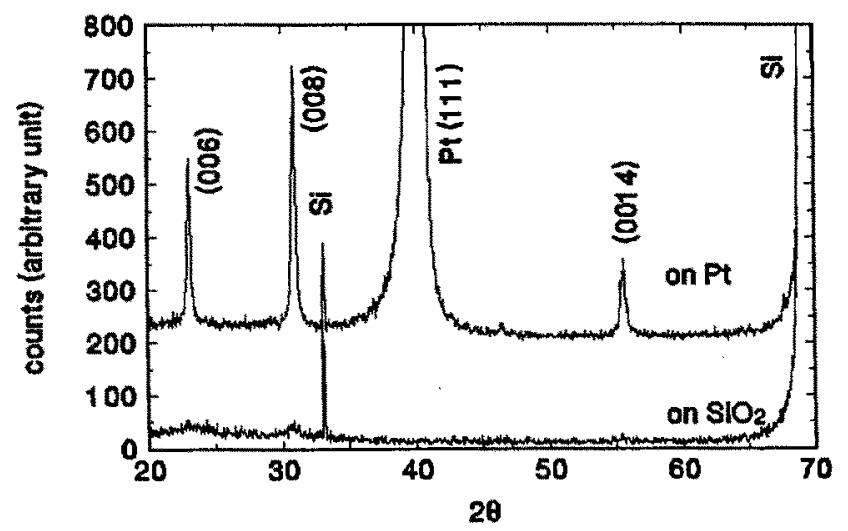

Fig. 8. X-ray diffraction patterns of two in-situ annealed films on $2000 \AA . \mathrm{Pt}$ underlayer, and on $\mathrm{SiO}_{2}$ substrate. The thickness of both films are $800 \AA$. 


\section{DISCUSSION AND CONCLUSION}

The experimental results suggest that there are two different types of nucleation during the ex-situ annealing process. The nucleation within the film "bulk" tends to form crystallites with random c-axis orientations, whereas the nucleation at the interface(s) with the substrates or underlayers forms crystallites with the c-axis predominantly perpendicularly oriented. The atoms deposited on the $\mathrm{Pt}$ underlayer may be arranged more easily into their lowest surface energy state. As a result, more sites with higher inplane short-range atomic order are formed at the Pt interface. The amorphous films which have in-plane short-range order were shown to become films with perpendicular c-axis orientation after annealing [7]. These sites are nucleation centers for grains with perpendicular c-axis orientation. The layers which are at a distance from the interface (or in the "bulk") may not have the in-plane short-range order and only have random short-range order. These sites are the nucleation centers for randomly oriented grains. The pre-anneal at 600 ${ }^{\circ} \mathrm{C}$ before crystallization at $790{ }^{\circ} \mathrm{C}$ or $810{ }^{\circ} \mathrm{C}$ appears to promote the surface nuclei more than the "bulk" nuclei, and change the distribution of nuclei in favor of perpendicular caxis orientation.

The barium ferrite films with low $\mathrm{Ba}$ content were shown to have low nucleation in the "bulk" [5]. It is, therefore, easier to obtain perpendicular orientation in these films when $\mathrm{Pt}$ underlayers are used. The low Ba content films which are 800 $\AA$ thick or even $1250 \AA$ with the two-step annealing are still perpendicularly oriented as indicated by the higher $S_{q}$ in the perpendicular direction. In contrast, the high $\mathrm{Ba}$ content films became almost randomly oriented at $500 \AA$, as suggested by the similar $\mathrm{M}_{\mathrm{r}} \mathrm{t}, \mathrm{H}_{\mathrm{c}}$ and $\mathrm{S}_{\mathrm{q}}$ values in perpendicular and inplane directions. In fact, $S_{q}$ values of $0.5 \sim 0.6$ as found in these films also indicated nearly 3-D random c-axis orientation [8]. The "bulk" nucleation can be dominant in high $\mathrm{Ba}$ content films due to the greatly enhanced nucleation rate and reduced growth rate, which, however, results in random c-axis orientation. In order to enhance surface nucleation in high $\mathrm{Ba}$ content films relative to the "bulk" nucleation, thus producing films with small grains and stronger perpendicular orientation, very thin film thickness and the two-step annealing process are required. Since the number of "bulk" nucleation sites decreases linearly with the film thickness, while the number of nucleation sites at the interface is relatively independent of the film thickness, nearly perfectly perpendicular orientation can be achieved in high Ba content films at the film thickness of $300 \AA$ using the two-step annealing.

Better perpendicular orientation can be obtained in $500 \AA$ thick low Ba content films on a $2000 \AA$ thick Pt underlayer than on thinner Pt underlayers. This is possibly due to the larger $\mathrm{Pt}$ grain size which may be more suitable for the surface nuclei of barium ferrite to form. The $500 \AA$ high Ba content films were not affected by the Pt underlayer thickness due to the predominant "bulk" nucleations in these films.
It was shown that incoherent rotation, which causes lower $H_{c}$ values than the anisotropy field $H_{k}$, is more dominant in the films with larger grain size [9]. In fact, the StonerWohlfarth coherent rotation model predicts that $\mathrm{H}_{\mathrm{c}}$ becomes equal to $H_{k}$ in a system with aligned easy axes [8]. Due to incoherent rotation, however, neither the $500 \AA$ low Ba content film A, nor the $300 \AA$ high Ba content film B, despite their excellent perpendicular orientations, have $H_{c}$ values nearly as high as $\mathrm{H}_{\mathbf{k}}$.

The grains whose c-axes are perpendicularly oriented are generally larger than those with random orientation. The grains with random $c$-axis orientation have one of the two fast grain growth directions in the ab plane restrained by the film thickness $[10,11]$. The grains with $c$-axis perpendicular to the film plane have both of their fast growth axes in-plane, and their growth will continue until the crystallites have completely encroached on one another. The average growth rate is, therefore, higher in the films with perpendicular c-axis orientation, and the grain size is larger. As a result, the change of film orientation from random to perpendicular usually causes a decrease in $\mathrm{H}_{\mathrm{c}}$ due to increased incoherent rotation and as seen in Fig. 3.

The films deposited on $\mathrm{SiO}_{2}$ suffer from interdiffusion with $\mathrm{Si}$, which may suppress nucleation in the diffused layer [12]. As compared to the films deposited on Pt underlayers, the films on $\mathrm{SiO}_{2}$ have lower $\mathrm{M}_{s}$, and thicker films are required for crystallization under the same annealing conditions.

Self-epitaxial growth may occur in films with in-situ annealing due to higher atom mobility [1]. The perpendicular surface film texture is maintained upon further film growth. Perpendicular orientions were thus observed in the in-situ annealed films even when $\mathrm{SiO}_{2}$ substrates were used without $\mathrm{Pt}$ underlayers. The lower value of $\mathrm{H}_{\mathrm{c}}$ in the high $\mathrm{Ba}$ content films under in-situ annealing than in those under ex-situ annealing could be related to the larger grain size [9], which may be due to higher atom mobility under in-situ annealing.

\section{REFERENCES}

[1] A. Morisako, M. Matsumoto and M. Naoe, IEEE Trans. Magn., vol. 23, pp. 56-58, 1987.

[2] X. Sui and M. H. Kryder, Appl. Phys. Lett., vol. 63, pp. 15821-23, 1993. [3]P. C. Dorsey, C. Vittoria, J. Magn. Magn. Mater., vol. 137, pp. 89-97, 1994.

[4] X. Sui, B. K. Cheong, D. E. Laughlin and M. H. Kryder, J. Magn. Soc. Jpn. vol. 18, no. S1, pp. 319-322, 1994

[5] Y. J. Chen, D. E. Laughlin, X. Ma, and M. H. Kryder, J. Appl. Phys., vol. 81, pp. 4380-4382, 1997.

[6] Y. J. Chen and M. H. Kryder, J. Appl. Phys., vol. 79, pp. 4878-4880, 1996.

[7] J. E. Snyder, V. G. Harris, N. C. Koon, X. Sui, and M. H. Kryder, IEEE Trans. Magn., vol. 31, pp. 3844-3846, 1995.

[8] E. C. Stoner, E. P. Wohlfarth, Phil. Trans. Roy. Soc. London, vol. A240, pp. 599-642, 1948.

[9] Y.J.Chen, W.Yang, D.N.Lambeth and M.H.Kryder, "Incoherent rotation in barium ferrite thin film recording media", IEEE Trans. Magn., in press.

[10] J. Smit and H. P. J. Wijn, Ferrites (Wiley, New York, 1959), Chap. 11.

[11] X. Sui, M. H. Kryder, B. Y. Wong and D. E. Laughlin, IEEE Trans. Magn., vol. 29, pp. 3751-3753, 1993.

[12] T. L. Hylton, M. A. Parker, K. R. Coffey, R. Umphress and J. K. Howard, J. Appl. Phys., vol. 75, pp. 5960-5965, 1994. 